\title{
Oocydin A, a chlorinated macrocyclic lactone with potent anti-oomycete activity from Serratia marcescens
}

\author{
Gary Strobel,, Jia-Yao Li, ${ }^{1}$ Fumio Sugawara, ${ }^{2}$ Hiroyuki Koshino, ${ }^{3}$ \\ James Harper ${ }^{4}$ and W. M. Hess ${ }^{5}$
}

Author for correspondence: Gary Strobel. Tel: +1 406994 5148. Fax: +1 4069947600. e-mail: uplgs@montana.edu

\footnotetext{
1 Department of Plant Sciences, Montana State University, Bozeman, MT 59717, USA

2 Department of Applied Biological Science, Science University of Tokyo, Noda, Chiba 278-8510, Japan

3 The Institute of Physical and Chemical Research, Wako, Saitama 351-0198, Tokyo, Japan

4 Department of Chemistry, University of Utah, Salt Lake City, UT 84112, USA

5 Department of Botany and Range Science, Brigham Young University, Provo, UT 84602, USA
}

\begin{abstract}
A unique chlorinated macrocyclic lactone, termed oocydin A, was isolated from a strain of Serratia marcescens growing as an epiphyte on Rhyncholacis pedicillata, an aquatic plant native to the Carrao river of the VenezuelanGuyanan region of South America. The lactone has a molecular mass of $470 \mathrm{Da}$, and contains one atom of chlorine, a carboxyl group and a tetrahydrofuran ring internal to a larger macrocyclic ring. MICs of approximately $0.03 \mu \mathrm{g} \mathrm{ml}^{-1}$ were noted for oocydin A against such phytopathogenic oomycetes as Pythium ultimum, Phytophthora parasitica, Phytophthora cinnamomi and Phytophthora citrophora. With regard to the true fungi, oocydin A had either minimal or no effect against certain Fungi Imperfecti (including several pathogens of humans), two ascomycetes and a basidiomycete. Oocydin A may have potential as an antimycotic in agricultural applications and especially for crop protection.
\end{abstract}

Keywords: antimycotic, phycomycetes, aquatic ecology, epiphyte

\section{INTRODUCTION}

Plants, especially those growing in freshwater environments, must have disease resistance mechanisms to cope with aquatic plant-pathogenic fungi that may invade and destroy them. Some of the most commonly known plant pathogens preferring aquatic environments are the oomycetes. This group of organisms (known as the water moulds) has recently been characterized as belonging to an entirely new biological kingdom known as the Stramenophila (Alexopoulos et al., 1996). The Stramenophila include such genera of phytopathogens as Phytophthora, Aphanomyces, Bremia, Pernospora, Pythium and Plasmopara. The presence of free water or high humidity is an absolute prerequisite for the pathogenicity of these organisms (Buczacki, 1983).

The oomycetes can be devastating to terrestrial plants; however, the appropriate moisture requirements must be met (Buczacki, 1983). Yet, conceivably these organisms could also attack and infect plants normally existing in strictly aquatic environments but nevertheless, aquatic plants seem to thrive and are relatively disease-free in their respective ecosystems. This biological conundrum may be related to either intrinsic or extrinsic factors controlling plant disease resistance, a phenomenon little understood or studied in aquatic plants. With regard to the possibility of extrinsic factors controlling disease, certain epiphytic or endophytic microbes may associate with aquatic plants and produce anti-oomycetous compounds. This may contribute to the defence of the plant by killing, inhibiting or warding off invading oomycetes. This rationale served as a basis for a search for microbes participating in associations with aquatic plants in which antimycotics may be produced by epiphytes or endophytes. If such compounds exist, they may prove agriculturally applicable to plant disease control situations in which one or more phytopathogenic oomycetes are a potential problem.

Rivers arising and flowing in the Venezuelan-Guyana of South America make up the region's primary bodies of fresh water. Rhyncholacis pedicillata is a small highly specialized aquatic plant of the family Podostemaceae that grows in colonies and thrives in some of the brownblack rivers of the Venezuelan-Guyana (Steyermark et al., 1995). These plant colonies are comprised of hundreds of individuals that grow firmly attached to rock surfaces and prefer swift water currents. $R$. pedicillata is $0.1-0.5 \mathrm{~m}$ in size and it has a slightly enlarged bulbous-like base and an extensive root system that anchors the plant to rock. Its stems are multibranched, are lace-like and covered with numerous small leaves. Close examination of individual plants in 
the Carrao river revealed animal or environmentally inflicted wounds on the stems. Normally, it would seem that such wounds would serve as entry points for one or more pathogenic oomycetes. However, little or no disease symptoms on the plants were observed.

Small stem pieces were examined for their associated microbes and each of those recovered was assayed against Pythium ultimum. The most commonly observed microbe was a strongly red-pigmented bacterium that was identified as Serratia marcescens. It colonized the surface of the stems of $R$. pedicillata, rather than internally. In culture, this bacterium produced a novel macrocyclic chlorinated lactone termed oocydin A that demonstrated selective toxicity (antimycotic) towards the oomycetes with extremely low MICs (e.g. 0.03 $\mu \mathrm{g} \mathrm{ml}^{-1}$ for various Phytophthora spp.). This report deals primarily with the isolation of $S$. marcescens and the chemical characterization of oocydin A. Some specific details are also given on the biological activity of the compound. Finally, its relationship to the biology of the plant-microbe interaction is discussed, along with its potential usefulness to agriculture.

\section{METHODS}

Isolation of S. marcescens and demonstration of antimycotic activity. Several small stem fragments of $R$. pedicillata were removed from the plant growing in its river environment and cut into pieces about $5.0 \mathrm{~mm}$ long. These tissue pieces were then soaked overnight in $0 \cdot 1 \mathrm{M}$ sodium phosphate buffer $(\mathrm{pH}$ $6 \cdot 8), 0 \cdot 1 \mathrm{M} \mathrm{NaCl}$. After $12 \mathrm{~h}$, the saline solution was collected, left at $23{ }^{\circ} \mathrm{C}$ for $1 \mathrm{~h}$ and then streaked onto a semi-selective medium (King's B; KB) according to the procedures previously outlined (Miller et al., 1998). After $2 \mathrm{~d}$, the most commonly appearing colonies consisted of bacilliform bacteria that produced a bright-red pigment. Each of these colonies was transferred back onto KB medium and incubated for several days prior to being transferred as individual colonies onto potato dextrose agar (PDA) plates. These plates were incubated for at least $3-5 \mathrm{~d}$ at $23^{\circ} \mathrm{C}$ prior to being co-inoculated (as $7 \times 7 \mathrm{~mm}$ infested agar plugs) with a rapidly growing culture of $P$. ultimum. The plates were then incubated at $23{ }^{\circ} \mathrm{C}$ and examined for antimycotic activity, detected as a zone of inhibition of fungal growth around the bacterial colony. The red-pigmented, antimycotic-producing bacterial strain was identified as $S$. marcescens by $\mathrm{Dr} J$. van der Toorn of the Identification Service of the Kluyver Laboratory for Biotechnology, Delft, The Netherlands. Our isolate of $S$. marcescens is stored in the bacterial culture collection of Montana State University, Bozeman, MT, USA, as acquisition no. 97 (MSU-97).

Fungal strains. All strains of Phytophthora used in bioassays were the generous gift of Dr John Menge, University of California, Riverside, CA, USA. P. ultimum and all other plant-pathogenic fungi used in bioassays were a generous gift of Dr Don Mathre, Montana State University. Standard isolates of $S$. marcescens, used for comparative purposes, were obtained from the Montana State University bacterial culture collection.

Bioassays. A simple plate bioassay was used to detect bioactivity of various fractions during the purification of oocydin A. Aliquots $(10-20 \mu \mathrm{l})$ of sample were placed on a PDA plate and dried. The plates were then inoculated with four $7 \times 7 \mathrm{~mm}$ plugs of agar infested with $P$. ultimum, one in each quadrant, and incubated for $36-48 \mathrm{~h}$ at $23^{\circ} \mathrm{C}$. Antimycotic activity was apparent as a zone of growth inhibition. The antimycotic activity of pure oocydin A was also tested against a series of plant pathogens by dissolving $50 \mu \mathrm{g}$ of the compound in $100 \mu \mathrm{l}$ methanol, spotting $10 \mu \mathrm{l}$ portions onto PDA plates and allowing the droplets to dry. Each plate was overlaid or sprayed with an aqueous suspension of the test fungus in water (containing mycelial fragments and/or spores), sealed with a piece of Parafilm and then incubated at $23{ }^{\circ} \mathrm{C}$ for $4-5 \mathrm{~d}$. In each case, a positive control test was also conducted (the residue obtained from the methylene-chlorideextracted medium without the bacterium). MICs of oocydin A against various phycomycetes were determined by preparing a stock solution of the compound $\left(1 \mathrm{mg} \mathrm{ml}^{-1}\right.$ in methanol). This was dispensed, by serial twofold dilution, into a 24 -well plate. Each well of the plate contained $500 \mu$ l potato dextrose (PD) broth. A small plug of the test fungus was placed into each well and the plate incubated for $48-72 \mathrm{~h}$ at $23^{\circ} \mathrm{C}$. The MIC was taken as the concentration of oocydin $\mathrm{A}$ in the well where no growth was detected visually after either 48 or $72 \mathrm{~h}$.

MIC tests were also conducted for several fungal pathogens of humans using the microbroth dilution assay as recommended by the subcommittee on antifungal susceptibility testing of the US National Committee for Clinical Laboratory Standards (NCCLS). The effects of the well-established antimycotics metalaxyl, pseudomycin B and amphotericin B were determined concomitantly.

Oocydin A was tested against human cancer cell lines BT-20, MCF 7 (both from ATCC, Manassas, VA, USA) and a normal human mammary cell line, cc2551 HMEC (from Clonetics, San Diego, CA, USA). These cells were exposed to serial dilutions of oocydin A. After $3 \mathrm{~d}$, they were stained with neutral red and the absorbance measured at $540 \mathrm{~nm}$. For nonadherent cells, a modification of the staining protocol was used (Berent et al., 1986). The results were recorded as median inhibitory concentrations ( $\mathrm{IC}_{50}$ values).

Oocydin A isolation procedure. Cells from a single colony of S. marcescens MSU-97 were used to inoculate $15 \mathrm{ml}$ PD broth and the culture was grown overnight with shaking at $23^{\circ} \mathrm{C}$. This culture was then used to inoculate two $2 \cdot 0$ litre flasks, each containing 11 of medium consisting of $24 \mathrm{~g} \mathrm{PD} ; 6 \mathrm{~g}$ soytone; $4 \mathrm{~g}$ yeast extract and $100 \mathrm{mg} \mathrm{NaCl}$ in $\mathrm{H}_{2} \mathrm{O}$. The cultures were incubated at $23{ }^{\circ} \mathrm{C}$ for $15 \mathrm{~d}$. The micro-organism was removed by centrifugation and the culture fluid extracted twice with two equal volumes of methylene chloride. This solvent was taken to dryness by flash evaporation at $45-50{ }^{\circ} \mathrm{C}$ under vacuum. Approximately $2.1 \mathrm{~g}$ of residue was present after evaporation of the solvent. This material was dissolved in chloroform $(5 \mathrm{ml})$ and placed on a $3.0 \times 20 \cdot 0 \mathrm{~cm}$ column of silica gel. The column was eluted with a chloroform/methanol $(10: 1, \mathrm{v} / \mathrm{v})$ solution. The first $100 \mathrm{ml}$ to elute from the column was discarded and the next $50-100 \mathrm{ml}$ contained the antimycotic activity. This material was placed onto another silica gel column (the same size as initially used) and eluted with methylene chloride/methanol $(10: 1, \mathrm{v} / \mathrm{v})$ solution. In this case, the first $50 \mathrm{ml}$ of eluate was discarded and the next $80 \mathrm{ml}$ contained the bioactivity (approx. 50-60 mg). The mixture of compounds was then subjected to a series of preparative TLC steps on $20 \times 20 \mathrm{~cm}$ plates to yield a pure product. The solvent systems used in succession were as follows: solvent $\mathrm{A}$, chloroform/methanol/acetic acid (12:1:0.1, by vol.); solvent $\mathrm{B}$, chloroform/methanol/ammonium hydroxide $(6: 2: 0 \cdot 1$, by vol.); and solvent C, methylene chloride/methanol $(6: 2, \mathrm{v} / \mathrm{v})$. After each successive TLC step, each band on the plate was eluted with methanol and the material possessing bioactivity 
(see bioassay procedure above) was reapplied to the next plate prior to separation. Once obtained and authenticated, a small amount of oocydin A was applied at the margin to the TLC plate as an appropriate reference for larger scale preparations. It appeared as a brownish spot with the vanillin-sulfuric reagent after gentle heating (Cardellina, 1991). Approximately 15-18 mg oocydin A was recovered per litre of culture fluid. It was obtained as a whitish powder by dissolving it in a methanolic-aqueous solution, which was then frozen and subjected to lyophilization. This was the preparation that was subjected to physical, chemical and biological characterization, once the purity of the sample was firmly established.

HPLC. The putative TLC-purified sample of oocydin A (20 $\mu \mathrm{g})$ was subjected to HPLC using an Altima C-18 column $(7.0 \times 250 \mathrm{~mm})$ (Alltech) and eluting with a linear gradient starting with a solution of $0 \cdot 1 \%$ TFA $/ 30 \%$ acetonitrile in water and finishing with a solution of $0 \cdot 1 \%$ TFA $/ 50 \%$ acetonitrile in water, over the course of $30 \mathrm{~min}$. Detection was performed with a Waters variable wavelength detector at $208 \mathrm{~nm}$.

Spectroscopic analyses. After the purity of oocydin A had been established it was subjected to a series of analyses using standard spectroscopic techniques. Electrospray mass spectroscopy was performed by dissolving the sample in methanol/water/acetic acid (50:50:1, by vol.). The sample was then injected into Montana State University's custombuilt mass spectrometer with a spray flow of $2 \mu \mathrm{l} \mathrm{min}{ }^{-1}$ and a spray voltage of $2 \cdot 2 \mathrm{kV}$ via the loop injection method. Oocydin A was also subjected to laser desorption mass spectroscopy on a Perspectives Biosystems instrument. The sample was incorporated into a matrix of 3,5-dihydroxybenzoic acid and scanned accordingly. The following parameters were used: accelerating voltage $30000 \mathrm{~V}$, grid voltage $70 \%$, mirror ratio $1 \cdot 06$, laser 260 and cytochrome $c$ to standardize the instrument. Negative high resolution FAB mass spectroscopy was done to acquire empirical formula data. Elemental analysis was done by degradative techniques at Atlantic Microlab, Norcross, GA, USA. Infrared spectroscopy was done on a Perkin Elmer instrument with oocydin A embedded in a matrix of anhydrous $\mathrm{KBr}$ and pressed into a pellet. An average of 16 scans was taken on the sample. The UV absorption spectrum of oocydin A was determined in $1 \mathrm{ml} 100 \%$ methanol $(1.0 \mathrm{~cm}$ light path, Beckman DU 50 spectrophotometer). Its optical rotation was determined in a sample dissolved in $100 \%$ methanol and analysed in a JASCO P1010 instrument.

Oocydin A was subjected to NMR techniques after being dissolved in $100 \%$ deuterated methanol. Initially, the ${ }^{1} \mathrm{H}$ spectrum was obtained on a Bruker DRX 500 instrument with 64 scans and a delay cycle of $2 \mathrm{~s}$, and collected as $32 \mathrm{k}$ real-time domain points using a transmitter frequency of $500 \cdot 13 \mathrm{MHz}$. The ${ }^{1} \mathrm{H}$ spectrum was referenced to the MeOD (deuterated methanol) signal at $3 \cdot 3$ p.p.m. For acquisition of the ${ }^{13} \mathrm{C}$ spectrum, 4096 scans were made with a recycle delay of $10 \mathrm{~s}$ and collected as $32 \mathrm{k}$ real-time domain points using a transmitter frequency of $125 \cdot 77 \mathrm{MHz}$. The spectrum was referenced to the residue MeOD signal at $49 \cdot 0$ p.p.m.

Oocydin A was also analysed by 2D INADEQUATE analysis on a $500 \mathrm{MHz}$ Varian Inova spectrometer operating at $125.892 \mathrm{MHz}$ and $26^{\circ} \mathrm{C}$. Oocydin A (58 mg) was dissolved in $200 \mu \mathrm{CDCl}_{3}$ and the analysis performed using a Varian $5 \mathrm{~mm}$ probe and a Shigemi microtube whose susceptibility was matched to the solvent used. Analysis parameters included a $10 \mu \mathrm{s}{ }^{13} \mathrm{C} 90^{\circ}$ degree pulse and a pulse sequence delay optimized for detection of $55 \mathrm{~Hz}$ carbon-carbon scalar coupling constants. A total of 64 evolution increments of 800 transients each were used for an analysis time of $4 \cdot 7 \mathrm{~d}$. Digital resolution of the acquisition and evolution dimensions were $0 \cdot 2$ and $176 \cdot 1 \mathrm{~Hz}$ per point, respectively. The gross structure of oocydin A was deduced from the various NMR data, particularly gradient DQF-COSY, gradient HMQC, gradient HMBC and difference 1D-NOE spectra in a JEOL JNM-alpha 600 spectrometer. Stereochemistry of oocydin A was primarily determined by $1 \mathrm{D}-\mathrm{NOE}$ and selective NOE experiments. INADEQUATE spectral processing and signal assignments were done in a near automated fashion using software described by Dunkel et al. (1990, 1992) and Harper et al. (1996).

Scanning electron microscopy. Materials to be examined were placed in $2 \%$ glutaraldehyde in $0 \cdot 1 \mathrm{M}$ sodium cacodylate buffer (pH 7.2-7·4) (Upadhyay et al., 1991). The samples were critical-point-dried, gold-coated with a sputter coater, and observed and photographed with a JEOL 6100 scanning electron microscope. Bacterial preparations were supported on dried $\gamma$-irradiated carnation leaves.

Materials. All solvents used for HPLC and TLC were HPLC grade. Solvents used for extraction of oocydin A were ACS grade. All TLC was conducted on EM-Merck precoated glass silica gel plates at a thickness of $0.25 \mathrm{~mm}$.

\section{RESULTS AND DISCUSSION}

\section{Identity, isolation and biology of the bacterium}

The bacterium (MSU-97) used in this study was identified as Serratia marcescens based on a series of biochemical tests conducted at the Kluyver Laboratory for Biotechnology, Delft, The Netherlands. This bacterium, based on its deep-red pigmentation, was the most commonly observed bacterial isolate obtained from $R$. pedicillata, an aquatic plant native to rapidly flowing freshwater rivers of the Venezuelan-Guayana region of South America (Fig. 1a). Examination of the surface of some small stems of $R$. pedicillata by scanning electron microscopy revealed that bacterial colonies were in abundance. Hundreds of individual cells seemed to constitute each colony (Fig. 1b). Individual bacterial cells also appeared on the plant surface. The identity of the bacteria could not be firmly attributed to $S$. marcescens by scanning electron microscopy, but the surface characteristics of the bacteria on the plant (Fig. 1c) were identical to those of bacteria in pure cultures of authenticated S. marcescens (isolate 97) (Figs 1c, d). These included multiple small projections and a generally relatively rough cell surface. Such surface characteristics do not appear to be common in bacteria and may serve as a means to help locate and identify $S$. marcescens on the surface of its host. In addition, the size and shape of the bacterial cells on the plant and in the authenticated culture appeared to be identical. This bacterium was labelled as MSU-97, and stored both in $15 \%$ glycerol and at $-70{ }^{\circ} \mathrm{C}$. Since $S$. marcescens was primarily recovered from the plant surface and not from internal tissues, it was considered more epiphytic than endophytic in its relationship to the plant.

\section{Purification of oocydin A from S. marcescens}

Several antimycotic-producing bacteria were isolated from $R$. pedicillata, but $S$. marcescens was by far the most prevalent. Plate bioassays demonstrated that $S$. 

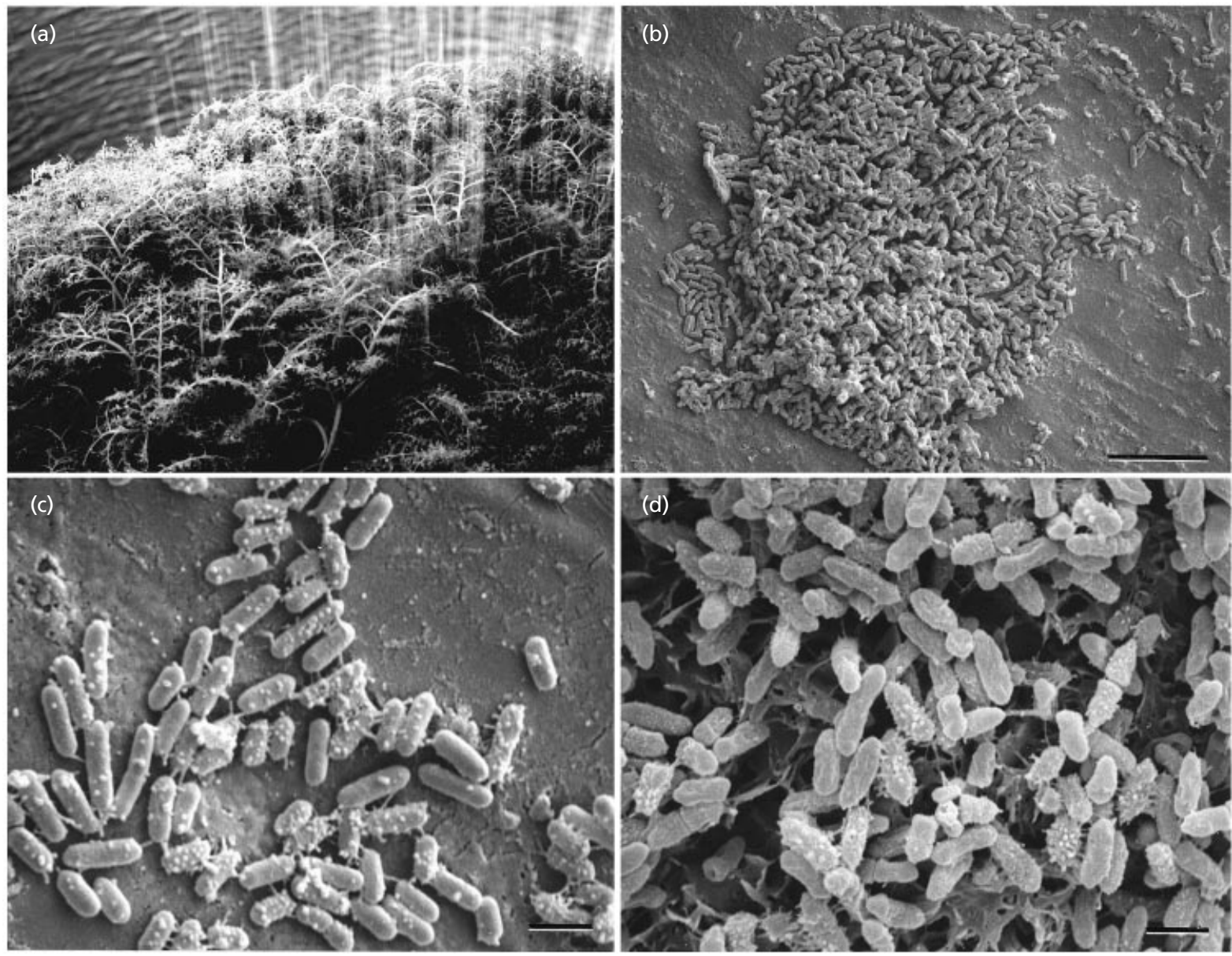

Fig. 1. S. marcescens and its relationship to $R$. pedicillata. (a) The plant ( $R$. pedicillata) from which $S$. marcescens was originally isolated growing beneath the Sappo waterfall in the Carrao river of Venezuela. (b) A scanning electron micrograph of a colony of $S$. marcescens attached to the surface of the stem of $R$. pedicillata. Bar, $10 \mu \mathrm{m}$. (c) A scanning electron micrograph at higher magnification of $S$. marcescens illustrating its size and surface characteristics on the plant surface. Bar, $1 \mu \mathrm{m}$. (d) The bacterium that was positively identified as $S$. marcescens grown in pure culture and then examined by scanning electron microscopy. Bar, $1 \mu \mathrm{m}$.

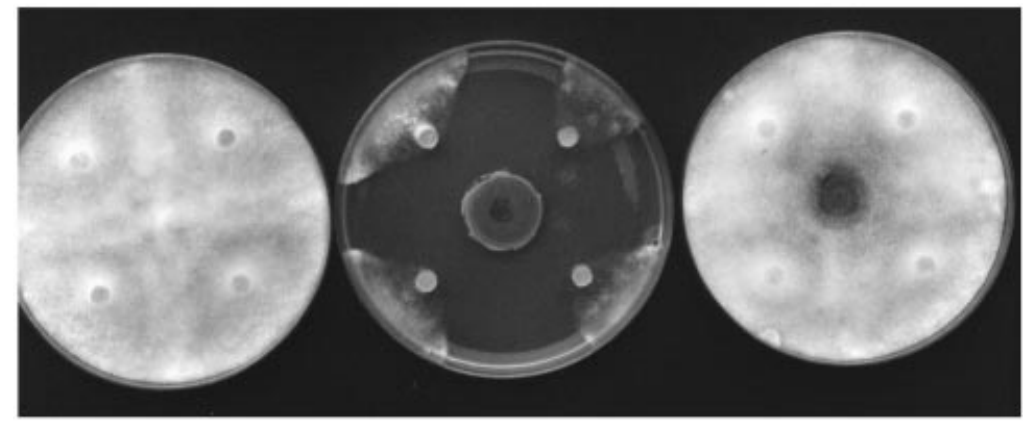

Fig. 2. Effect of $S$. marcescens MSU-97 (centre), MSU-69 (left) and ATCC 1009 (right) on $P$. ultimum $3 \mathrm{~d}$ after inoculation of plates with the oomycete.

marcescens MSU-97 is a potent inhibitor of growth of $P$. ultimum (Fig. 2). Inhibition zones persisted on the plates at $23{ }^{\circ} \mathrm{C}$ until the agar in the plate desiccated (after 4-6 weeks). Since $S$. marcescens is a relatively common micro-organism, standard isolates were also screened in the antifungal plate test. Neither ATCC 1009 nor MSU-
69 demonstrated any antifungal activity diffusing from the culture after $3 \mathrm{~d}$ of exposure to P. ultimum (Fig. 2). Following the purification procedure described in Methods, the antimycotic from $S$. marcescens was subjected to a series of chromatographic tests to check its purity. In solvent systems $\mathrm{A}$ and $\mathrm{B}$, the compound 


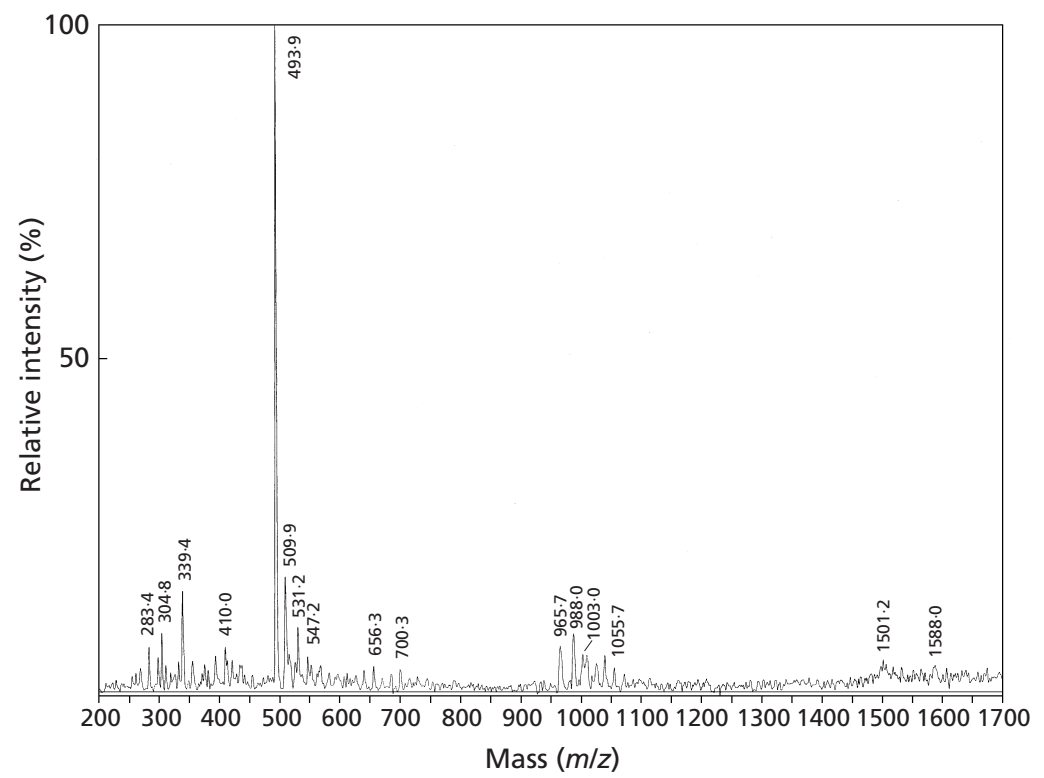

Fig. 3. The electrospray mass spectrum of oocydin A. The predominant peak at 493.9 is assigned to $(M+N a)^{+}$. appeared at $R_{\mathrm{F}}$ values of 0.49 and $0 \cdot 34$, respectively. In solvents consisting of methylene chloride/methanol $(6: 2, \mathrm{v} / \mathrm{v})$ and chloroform/methanol $(7: 1, \mathrm{v} / \mathrm{v})$, the $R_{\mathrm{F}}$ values were 0.65 and 0.27 , respectively. In each case, after spraying with the vanillin-sulfuric acid reagent, only one spot appeared on the plate, which was coincident with antimycotic activity. Furthermore, no other UV-absorbing or UV-fluorescent spots appeared on the plates after examination using long- or shortwavelength UV. After HPLC analyses, under the conditions described, only one sharp symmetrical peak absorbing at $208 \mathrm{~nm}$ appeared and it had a retention time of $19.7 \mathrm{~min}$. When the fractions corresponding to this peak were combined and subjected to the bioassay test the pool of fractions inhibited the growth of P. ultimum. Collectively, these chromatographic data suggested that the antimycotic agent of $S$. marcescens MSU-97 was homogeneous and ready for spectroscopic and chemical analyses.

\section{Characterization of oocydin A}

The uncorrected melting point of oocydin A was $106-108{ }^{\circ} \mathrm{C}$. The optical rotation, measured on $2 \cdot 2 \mathrm{mg}$ of the compound dissolved in $1 \mathrm{ml}$ methanol at $589 \mathrm{~nm}$ was $+18 \cdot 2^{\circ}$.

Electrospray mass spectroscopic analysis of oocydin A produced a major peak at $493.9 \mathrm{~m} / z$. This is consistent with a single charged species of $(M+\mathrm{Na})^{+}$(Fig. 3). The laser desorption spectrum also produced a major peak at 493.3 consistent with $(M+N a)^{+}$. However, there was also a significant $M+2$ peak at $495 \cdot 3$, which could be accounted for by oocydin A possessing a chlorine atom. This may be true because of a high abundance of the isotope ${ }^{37} \mathrm{Cl}$. Negative HRFAB data yielded $(M-\mathrm{H})^{+}$of $469 \cdot 1640$, which accounted for the empirical formula $\mathrm{C}_{23} \mathrm{H}_{30} \mathrm{O}_{8} \mathrm{Cl}_{1}$. The difference between the negative HRFAB data $\left[469=(M-H)^{+}\right]$and the electrospray laser desorption data $\left[M=(470+\mathrm{Na})^{+}\right]$is equivalent to $23 \mathrm{~m} / z$, which is the atomic mass of sodium. Therefore, in both the electrospray and laser desorption mass spectral analysis, oocydin A sequestered a sodium ion, which accounted for its molecular mass plus $23 \mathrm{~m} / \mathrm{z}$. In addition, the molecule assumed a net positive charge by picking up $\mathrm{H}^{+}$. Elemental analysis of oocydin $\mathrm{A}$ for its halogen content revealed that it was $=7 \cdot 1 \%$ (the expected $\mathrm{Cl}$ content is $7 \cdot 4 \%$ ). In the elemental analysis, the halogen was not specifically identified as chlorine. Although the negative HRFAB and the laser desorption mass spectral data gave a strong indication for the presence of chlorine in oocydin $\mathrm{A}$, these analyses were not totally definitive. Therefore, further supportive evidence was obtained with an Oxford Instrument energy-dispersive X-ray microanalysis system on a JEOL scanning electron microscope. The test was conducted on about $15-20 \mu \mathrm{g}$ oocydin A supported on an Al stub. A peak distinctive for chlorine and no other halogen appeared in the spectrum. Thus, the empirical formula for oocydin $\mathrm{A}$ is $\mathrm{C}_{23} \mathrm{H}_{31} \mathrm{O}_{8} \mathrm{Cl}_{1}$.

The chemical structure of oocydin A was ascertained by IR and NMR spectroscopic analyses since it was impossible, in our hands, to crystallize this substance and obtain X-ray analytical data. The IR absorption band at $2982 \mathrm{~cm}^{-1}$ with a shoulder suggested alkane and alkene functionalities, whereas the intense band at $1713 \mathrm{~cm}^{-1}$ indicated the presence of one or more acetate esters (Silverstein et al., 1991). Weaker bands at 1417 and $1602 \mathrm{~cm}^{-1}$ suggested the presence of a carboxylate functionality. Other notable bands occurred at 1640, $1124,1030,902$ and $835 \mathrm{~cm}^{-1}$. Although the IR spectrum was not that informative, it provided a data set unique to oocydin A and it did suggest the presence of certain functional groups.

The ${ }^{1} \mathrm{H}-\mathrm{NMR}$ spectrum was consistent with a compound having methyl, methylene and hydrogen bonded 


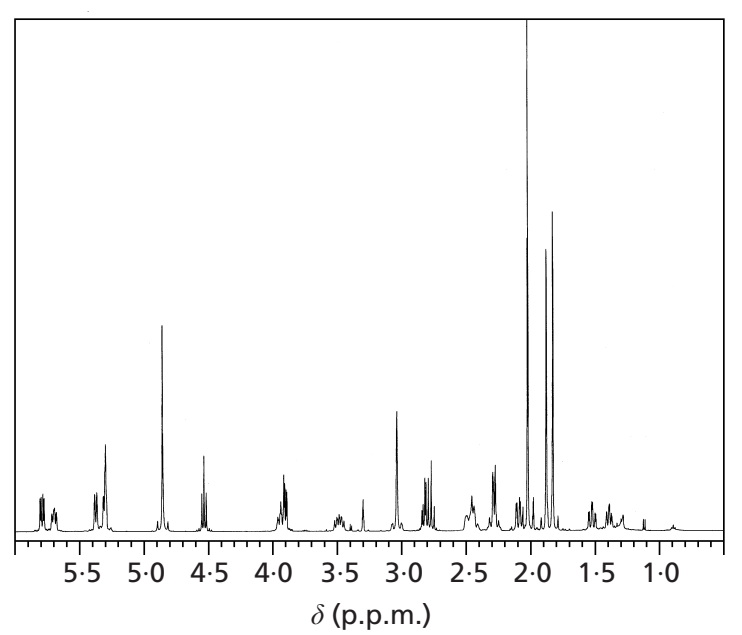

Fig. 4. The ${ }^{1} \mathrm{H}-\mathrm{NMR}$ spectrum of oocydin $\mathrm{A}$ obtained in $100 \%$ deuterated methanol. The peak at 3.3 p.p.m. is the $\mathrm{CD}_{3} \mathrm{OH}$ signal.

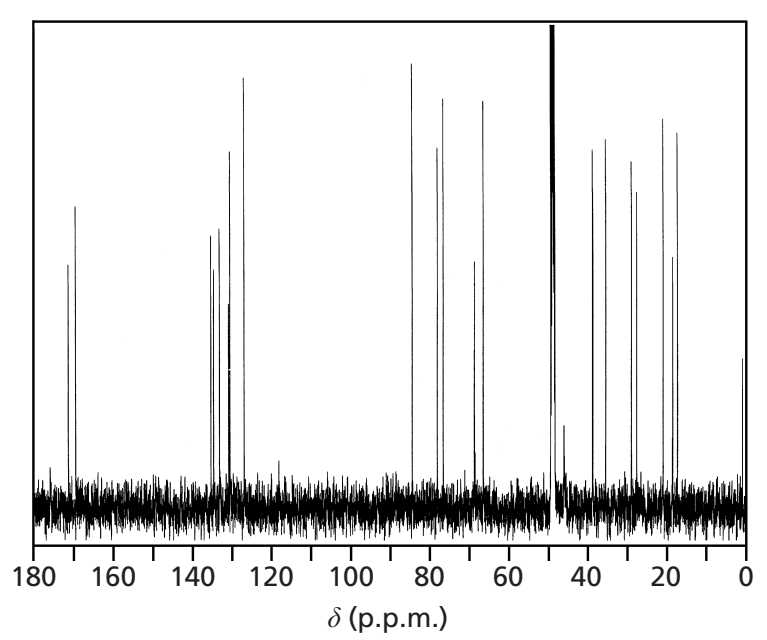

Fig. 5. The ${ }^{13} \mathrm{C}-\mathrm{NMR}$ spectrum of oocydin A obtained in $100 \%$ deuterated methanol. The signal at 49 p.p.m. is from the solvent carbon atom.

on carbons bearing oxygen (Fig. 4). In the ${ }^{13} \mathrm{C}-\mathrm{NMR}$ spectrum, it was possible to account for 23 carbon atoms (Fig. 5). This NMR analysis also showed doublebonded carbons, carbons bearing carbonyl groups and methyl carbons.

Whilst the one-dimensional NMR spectra of oocydin A were instructive, the structure proposed for this compound is primarily based on the use of a collection of sophisticated NMR techniques, each of which provided useful information on the relative positions of the atoms making up this compound. The carbon types were first determined by DEPT analysis (Doddrell et al., 1982). One bond and longer proton-carbon connections were established by HMQC and HMBC (Bax et al., 1983;

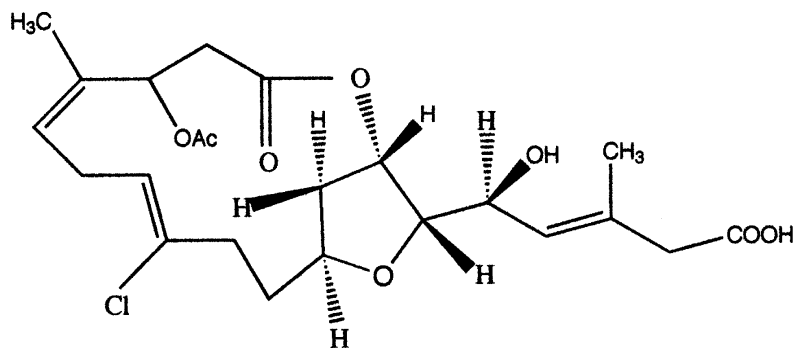

Fig. 6. The structure of oocydin A (no absolute stereochemistry is implied).

Bax \& Summers, 1986). Selective ROESY and NOE difference analyses allowed the relative stereochemical orientations to be established. A 2D INADEQUATE analysis was used to verify the proposed carbon-carbon connections (Bax et al., 1980, 1981). All INADEQUATE connections were determined using previously described data analysis software specifically suited to low signalto-noise acquisitions (Dunkel et al., 1990, 1992; Harper et al., 1996). The INADEQUATE analysis confirmed all but three bonds at greater than a $99 \cdot 9 \%$ confidence level. Thus, this very powerful, but relatively insensitive experimental technique confirmed all of the structural conclusions. A more complete description of the NMR analysis of oocydin A will be given elsewhere. Thus, oocydin A is a chlorinated macrocyclic lactone having a tetrahydrofuran ring internal to it with a side chain terminated by a carboxylic acid functionality. The proposed structure (Fig. 6) is consistent with all of the spectral information obtained. Although several asymmetric centres are present in the molecule, only the relative stereochemistry is presented and no absolute stereochemistry is implied. Oocydin A is one of only a few macrocyclic lactones that have ever been isolated. Others, including the fijinolides and the laulimalides, have been isolated from marine animals and demonstrate general toxic activity to various biological systems.

\section{Bioactivities of oocydin A}

The initial biological screen test showed the potent antimycotic activity of some substance(s) diffusing from the colonies of $S$. marcescens (Fig. 2). At least one bioactive substance that seemed to be involved in this phenomenon of fungal inhibition is oocydin A. The effect of purified oocydin A on several oomycetes was ascertained. It appeared that Phytophthora spp. were the most sensitive, with MICs of approximately $0.03 \mu \mathrm{g}$ $\mathrm{ml}^{-1}$ (Table 1). P. ultimum, however, was less sensitive than the Phytophthora spp. When tested on PDA plates $(5 \cdot 0 \mu \mathrm{g}$ oocydin A applied to a localized agar surface in a droplet of methanol) against a group of organisms representing the other three classes of fungi, oocydin A showed little or no activity. For instance, against Geotrichum candidum (Fungi Imperfecti) it was inactive; likewise only slight inhibition of growth was 
Table 1. The effects of oocydin A on various phytopathogenic oomycetes

MIC values were determined for representative oomycetes using diluted purified oocydin A in 24 well plates. A small plug of PDA containing the appropriate micro-organism was added to each well. The plates were incubated for $72 \mathrm{~h}$ and evaluated for growth of the test organism (see Methods).

\begin{tabular}{|c|c|c|c|}
\hline \multirow[t]{2}{*}{ Oomycete tested } & \multicolumn{2}{|c|}{$\begin{array}{l}\text { MIC of oocydin A } \\
\left(\mu \mathrm{g} \mathrm{ml}^{-1}\right)\end{array}$} & \multirow{2}{*}{$\begin{array}{c}\text { MIC of } \\
\text { metalaxyl } \\
\left(\mu \mathrm{g} \mathrm{ml}^{-1}\right)\end{array}$} \\
\hline & Test 1 & Test 2 & \\
\hline Pythium ultimum & $0 \cdot 11$ & $0 \cdot 39$ & ND \\
\hline Phytophthora parasitica & $0 \cdot 03$ & $0 \cdot 05$ & $0 \cdot 06$ \\
\hline Phytophthora cinnamomi & $0 \cdot 03$ & $0 \cdot 02$ & 0.06 \\
\hline Phytophthora citrophora & $0 \cdot 03$ & $0 \cdot 02$ & $\mathrm{ND}$ \\
\hline
\end{tabular}

ND, Not determined.

observed for Sclerotinia sclerotiorum (Ascomycetes) and Rhizoctonia solani (Basidiomycetes). In other plate tests, no activity was noted against either Verticillum dabliae or Alternaria tomato.

Oocydin A was tested against several important fungal pathogens of humans using the MIC microwell plate assay. At concentrations up to $80 \mu \mathrm{g} \mathrm{m}^{-1}$, it had no effect on Candida albicans, Cryptococcus neoformans, Aspergillus fumigatus, Candida parapsilosis or Histoplasma capsulatum. However, pseudomycin B inhibited growth of all of these organisms except A. fumigatus with MICs of $<1.0 \mu \mathrm{g} \mathrm{ml} l^{-1}$ and amphotericin B inhibited them all with MICs of $<1 \cdot 0 \mu \mathrm{g} \mathrm{ml}^{-1}$.

\section{Other biological considerations}

Oocydin A is a novel bioactive compound possessing some unique biological and chemical properties. Overall, it appears that it has selective lethal activity against oomycetes at MICs lower or similar to those noted for metalaxyl (Table 1). Metalaxyl is well established as the fungicide to which the oomyctes are the most sensitive (Lyr, 1995). However, in the past 10 years, increasing resistance to metalaxyl is developing in certain oomycete populations and this fungicide is being rendered useless or less effective (Lyr, 1995). Thus, a tremendous need exists for new compounds to combat these plant pathogens. These observations suggest that oocydin A may have potential as a novel antimycotic against some of the most important plant pathogens - the oomycetes. The cursory examination of the biological selectivity of oocydin A described here suggests that it targets one or more unique structural sites in the oomycetes. However, no mode of action studies on the compound have yet been done to confirm this suggestion. Oocydin A appears to have selective toxicity against various human cell lines. In preliminary studies, $\mathrm{IC}_{50}$ values of $0 \cdot 2 \mu \mathrm{g} \mathrm{ml} \mathrm{m}^{-1}$ against BT-20 (breast cancer cell line), $0.42 \mu \mathrm{g} \mathrm{ml} l^{-1}$ against MCF-7 (breast cancer cell line) and $0.6 \mu \mathrm{g} \mathrm{ml}^{-1}$ against a normal mammary cell line were noted. In future it may be possible to chemically modify oocydin A so that the compound is more selectively toxic to cancer cells. This would increase the likelihood that an oocydin A derivative could be more seriously considered as an anticancer drug candidate. However, a more immediate goal should be the exploration of oocydin A as a candidate compound for agricultural applications. This would necessitate the acquisition of large-scale amounts of the compound for plant testing and perhaps chemical modification to reduce any toxicity problems that may arise if the compound is to be used in controlling diseases of crops caused by oomycetes.

In natural settings, $S$. marcescens may play a role in the $R$. pedicillata relationship by first establishing itself on the plant surface and then producing oocydin A. This may eventually diffuse into the plant and/or, due to its solubility and charge characteristics, it may adhere to the plant surface. The presence of oocydin $\mathrm{A}$ in the area of the bacterial colony may prevent oomyceteous pathogens from causing rot or decay of the plant by virtue of the selective toxicity of this novel compound.

\section{ACKNOWLEDGEMENTS}

The authors greatly appreciate the invaluable help of Dr Concepcion Miller in isolating epiphytic bacteria, establishing bioassay systems and carrying out initial oocydin A separations. Dr Joe Sears of the Chemistry Department, Montana State University, kindly performed some of the mass spectral analyses. Dr Scott Busse of that same department did many of the NMR experiments. Mr Mike Standing of Brigham Young University, Provo, UT, helped to acquire the scanning electron micrographs. Dr Doug Zechner and Mike Rodriguez of Eli Lilly Co., Indianapolis, IN, gathered data on the effects of oocydin A and other antimycotics on many human fungal pathogens. Dr Richard Torczynski of Cytoclonal Pharmaceutics, Dallas, TX, kindly performed the human cell line tests. Dr Don Mathre of the Department of Plant Sciences, Montana State University, supplied metalaxyl.

\section{REFERENCES}

Alexopoulos, C. J., Mims, C. W. \& Strobel, G. A. (1996). Introductory Mycology, 4th edn. New York: Wiley.

Bax, A. \& Summers, M. F. (1986). ${ }^{1} \mathrm{H}$ and ${ }^{13} \mathrm{C}$ assignments from sensitivity-enhanced detection of heteronuclear multi-bond connectivity by $1 \mathrm{D}$ multiquantum NMR. J Am Chem Soc 108, 2093-2094.

Bax, A., Freeman, R. \& Kempsell, S. P. (1980). Natural abundance ${ }^{13} \mathrm{C}-{ }^{13} \mathrm{C}$ coupling observed via double quantum coherence. $\mathrm{J} \mathrm{Am}$ Chem Soc 102, 4849-4851.

Bax, A., Freeman, R. \& Frenkiel, T. A. (1981). An NMR technique for tracing out the carbon skeleton of an organic molecule. J Am Chem Soc 103, 2102-2104.

Bax, A., Griffey, R. H. \& Hawkins, B. L. (1983). Sensitivityenhanced correlation of ${ }^{15} \mathrm{~N}$ and ${ }^{1} \mathrm{H}$ chemical shifts in natural abundance samples via multiquantum coherence. J Am Chem Soc 105, 7188-7190.

Berent, S. L., Torczynski, R. M. \& Bollon, A. P. (1986). Sendai virus induces high levels of tumour necrosis factor mRNA in human peripheral blood leukocytes. Nucleic Acid Res 14, 8997-9015. 
Buczacki, S. T. (1983). Zoosporic Plant Pathogens. London: Academic Press.

Cardellina, J. H. (1991). HPLC separation of taxol and cephalomannine. J Chromatogr 14, 659-665.

Doddrell, D. M., Pegg, D. T. \& Bendall, M. R. (1982). Distortionless enhancement of NMR signal by polarization transfer. J Magn Reson 48, 323-327.

Dunkel, R., Mayne, C. L., Curtis, J., Pugmire, R. J. \& Grant, D. M. (1990). Computerized analysis of 2D INADEQUATE spectra. $J$ Magn Reson 90, 290-302.

Dunkel, R., Mayne, C. L., Pugmire, R. J. \& Grant, D. M. (1992). Improvements in the computerized analysis of 2D INADEQUATE spectra. Anal Chem 62, 3133-3149.

Harper, J. K., Dunkel, R., Wood, S. G., Owen, N. L., Li, D., Cates, R. G. \& Grant, D. M. (1996). NMR characterization of obscurinevine and obsurinevidine using novel computerized analysis techniques. J Chem Soc Perkin Trans I 2, 91-100.
Lyr, H. (1995). Modern Selective Fungicides. New York \& Jena: Gustav Fischer Verlag.

Miller, C. M., Miller, R. V., Garton-Kenny, D., Redgrave, B., Sears, J., Condron, M. M., Teplow, D. B. \& Strobel, G. A. (1998). Ecomycins, unique antimycotics from Pseudomonas viridiflava. J Appl Microbiol 84, 937-944.

Silverstein, R. M., Bassler, G. C. \& Morrill, T. C. (1991). Spectrometric Identification of Organic Compounds. New York: Wiley.

Steyermark, J. A., Berry, P. E. \& Holst, B. K. (1995). Flora of the Venezuelan Guayana. Portland, OR: Timber Press.

Upadhyay, R. V., Strobel, G. A. \& Hess, W. M. (1991). Morphogenesis and ultrastructure of the conidiomata of Aschochyta cypericola. Mycol Res 95, 785-791.

Received 20 April 1999; revised 4 August 1999; accepted 27 August 1999. 